Homology, Homotopy and Applications, vol.21(1), 2019, pp.341-350

\title{
A CANONICAL LIFT OF FROBENIUS IN MORAVA E-THEORY
}

\author{
NATHANIEL STAPLETON
}

(communicated by Emily Riehl)

\begin{abstract}
We prove that the $p$ th Hecke operator on the Morava $E$ cohomology of a space is congruent to the Frobenius mod $p$. This is a generalization of the fact that the $p$ th Adams operation on the complex $K$-theory of a space is congruent to the Frobenius mod $p$. The proof implies that the $p$ th Hecke operator may be used to test Rezk's congruence criterion.
\end{abstract}

\section{Introduction}

The $p$ th Adams operation on the complex $K$-theory of a space is congruent to the Frobenius $\bmod p$. This fact plays a role in Adams and Atiyah's proof [AA66] of the Hopf invariant one problem. It also implies the existence of a canonical operation $\theta$ on $K^{0}(X)$ satisfying

$$
\psi^{p}(x)=x^{p}+p \theta(x),
$$

when $K^{0}(X)$ is torsion-free. This extra structure was used by Bousfield [Bou96] to determine the $\lambda$-ring structure of the $K$-theory of an infinite loop space. There are several generalizations of the $p$ th Adams operation in complex $K$-theory to Morava $E$-theory: the $p$ th additive power operation, the $p$ th Adams operation, and the $p$ th Hecke operator. In this note, we show that the $p$ th Hecke operator is a lift of Frobenius.

In [Rez09], Rezk studies the relationship between two algebraic structures related to power operations in Morava $E$-theory. One structure is a monad $\mathbb{T}$ on the category of $E_{0}$-modules that is closely related to the free $E_{\infty}$-algebra functor. The other structure is a form of the Dyer-Lashof algebra for $E$, called $\Gamma$. Given a $\Gamma$-algebra $R$, each element $\sigma \in \Gamma$ gives rise to a linear endomorphism $Q_{\sigma}$ of $R$. He proves that a $\Gamma$-algebra $R$ admits the structure of an algebra over the monad $\mathbb{T}$ if and only if there exists an element $\sigma \in \Gamma$ (over a certain element $\bar{\sigma} \in \Gamma / p$ ) such that $Q_{\sigma}$ is a lift of Frobenius in the following sense:

$$
Q_{\sigma}(r) \equiv r^{p} \bmod p R
$$

for all $r \in R$.

We will show that $Q_{\sigma}$ may be taken to be the $p$ th Hecke operator $T_{p}$ as defined by Ando in [And95, Section 3.6]. We prove this by producing a canonical element $\sigma_{\text {can }} \in$ $\Gamma$ lifting the Frobenius class $\bar{\sigma} \in \Gamma / p\left[\mathbf{R e z 0 9}\right.$, Section 10.3] such that $Q_{\sigma_{c a n}}=T_{p}$. This

Received May 26, 2016, revised October 28, 2016, September 4, 2018; published November 7, 2018. 2010 Mathematics Subject Classification: 55N20.

Key words and phrases: Morava E-theory, Frobenius, Hecke operator.

Article available at http://dx.doi.org/10.4310/HHA.2019.v21.n1.a16

Copyright (c) 2018, Nathaniel Stapleton. Permission to copy for private use granted. 
provides us with extra algebraic structure on torsion-free algebras over the monad $\mathbb{T}$ in the form of a canonical operation $\theta$ satisfying

$$
T_{p}(r)=r^{p}+p \theta(r) .
$$

Let $\mathbb{G}_{E_{0}}$ be the formal group associated to $E$, a Morava $E$-theory spectrum. The Frobenius $\phi$ on $E_{0} / p$ induces the relative Frobenius isogeny

$$
\mathbb{G}_{E_{0} / p} \longrightarrow \phi^{*} \mathbb{G}_{E_{0} / p}
$$

over $E_{0} / p$. The kernel of this isogeny is a subgroup scheme of order $p$. By a theorem of Strickland, this corresponds to an $E_{0}$-algebra map

$$
\bar{\sigma}: E^{0}\left(B \Sigma_{p}\right) / I \longrightarrow E_{0} / p
$$

where $I$ is the image of the transfer from the trivial group to $\Sigma_{p}$. This map further corresponds to an element in the $\bmod p$ Dyer-Lashof algebra $\Gamma / p$. Rezk considers the set of $E_{0}$-module maps $[\bar{\sigma}] \subset \operatorname{hom}\left(E^{0}\left(B \Sigma_{p}\right) / I, E_{0}\right)$ lifting $\bar{\sigma}$.

Proposition 1.1. There is a canonical choice of lift $\sigma_{\text {can }} \in[\bar{\sigma}]$.

The construction of $\sigma_{c a n}$ is an application of the formula for the $K(n)$-local transfer (induction) along the surjection from $\Sigma_{p}$ to the trivial group [Gan06, Section 7.3].

Let $X$ be a space and let

$$
P_{p} / I: E^{0}(X) \longrightarrow E^{0}\left(B \Sigma_{p}\right) / I \otimes_{E_{0}} E^{0}(X)
$$

be the $p$ th additive power operation. The endomorphism $Q_{\sigma_{c a n}}$ of $E^{0}(X)$ is the composite of $P_{p} / I$ with $\sigma_{c a n} \otimes 1$.

Proposition 1.2. For any space $X$, the following operations on $E^{0}(X)$ are equal:

$$
Q_{\sigma_{c a n}}=\left(\sigma_{c a n} \otimes 1\right)\left(P_{p} / I\right)=T_{p} .
$$

This has the following immediate consequence:

Corollary 1.3. Let $X$ be a space such that $E^{0}(X)$ is torsion-free. There exists a canonical operation

$$
\theta: E^{0}(X) \longrightarrow E^{0}(X)
$$

such that, for all $x \in E^{0}(X)$,

$$
T_{p}(x)=x^{p}+p \theta(x) .
$$

\section{Acknowledgments}

It is a pleasure to thank Tobias Barthel, Charles Rezk, Tomer Schlank, and Mahmoud Zeinalian for helpful discussions and to thank the Max Planck Institute for Mathematics for its hospitality.

\section{Tools}

Let $E$ be a height $n$ Morava $E$-theory spectrum at the prime $p$. We will make use of several tools that let us access $E$-cohomology. We summarize them in this section. 
For the remainder of this paper, let $E(X)=E^{0}(X)$ for any space $X$. We will also write $E$ for the coefficients $E^{0}$ unless we state otherwise.

Character theory: Hopkins, Kuhn, and Ravenel introduce character theory for $E(B G)$ in [HKR00]. They construct the rationalized Drinfeld ring $C_{0}$ and introduce a ring of generalized class functions taking values in $C_{0}$ :

$C l_{n}\left(G, C_{0}\right)=\left\{C_{0}\right.$-valued functions on conjugacy classes of map from $\mathbb{Z}_{p}^{n}$ to $\left.G\right\}$.

They construct a map

$$
E(B G) \longrightarrow C l_{n}\left(G, C_{0}\right)
$$

and show that it induces an isomorphism after the domain has been base-changed to $C_{0}$ [HKR00, Theorem $\left.\mathrm{C}\right]$. When $n=1$, this is a $p$-adic version of the classical character map from representation theory.

Good groups: A finite group $G$ is good if the character map

$$
E(B G) \longrightarrow C l_{n}\left(G, C_{0}\right)
$$

is injective. Hopkins, Kuhn, and Ravenel show that $\Sigma_{p^{k}}$ is good for all $k$ [HKR00, Theorem 7.3].

Transfer maps: It follows from a result of Greenlees and Sadofsky [GS96] that there are transfer maps in $E$-cohomology along all maps of finite groups. In [Gan06, Section 7.3], Ganter studies the case of the transfer from $G$ to the trivial group and shows that there is a simple formula for the transfer on the level of class functions. Let

$$
\operatorname{Tr}_{C_{0}}: C l_{n}\left(G, C_{0}\right) \rightarrow C_{0}
$$

be given by the formula $f \mapsto \frac{1}{|G|} \sum_{\alpha} f(\alpha)$, where the sum runs over all of the maps $\alpha: \mathbb{Z}_{p}^{n} \rightarrow G$. Ganter shows that there is a commutative diagram

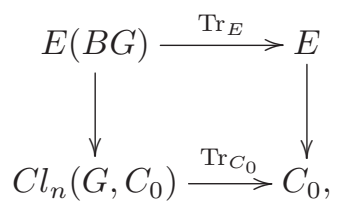

in which the vertical maps are the character map.

Subgroups of formal groups: Let $\mathbb{G}_{E}=\operatorname{Spf}\left(E\left(B S^{1}\right)\right)$ be the formal group associated to the spectrum $E$. In [Str98], Strickland produces a canonical isomorphism

$$
\operatorname{Spf}\left(E\left(B \Sigma_{p^{k}}\right) / I\right) \cong \operatorname{Sub}_{p^{k}}\left(\mathbb{G}_{E}\right),
$$

where $I$ is the image of the transfer along $\Sigma_{p^{k-1}}^{\times p} \subset \Sigma_{p^{k}}$ and $\operatorname{Sub}_{p^{k}}\left(\mathbb{G}_{E}\right)$ is the scheme that classifies subgroup schemes of order $p^{k}$ in $\mathbb{G}_{E}$. We will only need the case $k=1$.

The Frobenius class: The relative Frobenius is a degree $p$ isogeny of formal groups

$$
\mathbb{G}_{E / p} \rightarrow \phi^{*} \mathbb{G}_{E / p}
$$

where $\phi: E / p \rightarrow E / p$ is the Frobenius. The kernel of the map is a subgroup scheme of order $p$. Using Strickland's result, there is a canonical map of $E$-algebras

$$
\bar{\sigma}: E\left(B \Sigma_{p}\right) / I \rightarrow E / p
$$

picking out the kernel. In [Rez09, Section 10.3], Rezk describes this map in terms 
of a coordinate and considers the set of $E$-module maps $[\bar{\sigma}] \subset \operatorname{hom}\left(E\left(B \Sigma_{p}\right), E\right)$ that lift $\bar{\sigma}$.

Power operations: In [GH04], Goerss, Hopkins, and Miller prove that the spectrum $E$ admits the structure of an $E_{\infty}$-ring spectrum in an essentially unique way. This implies a theory of power operations. These are natural multiplicative non-additive maps

$$
P_{m}: E(X) \rightarrow E\left(B \Sigma_{m}\right) \otimes_{E} E(X)
$$

for all $m>0$. For $m=p^{k}$, they can be simplified to obtain interesting ring maps by further passing to the quotient

$$
P_{p^{k}} / I: E(X) \rightarrow E\left(B \Sigma_{p^{k}}\right) \otimes_{E} E(X) \rightarrow E\left(B \Sigma_{p^{k}}\right) / I \otimes_{E} E(X),
$$

where $I$ is the transfer ideal that appeared above.

Hecke operators: In [And95, Section 3.6], Ando produces operations

$$
T_{p^{k}}: E(X) \rightarrow E(X)
$$

by combining the structure of power operations, Strickland's result, and ideas from character theory. Let $\mathbb{T}=\left(\mathbb{Q}_{p} / \mathbb{Z}_{p}\right)^{n}$, let $H \subset \mathbb{T}$ be a finite subgroup, and let $D_{\infty}$ be the Drinfeld ring at infinite level so that $\operatorname{Spf}\left(D_{\infty}\right)=\operatorname{Level}\left(\mathbb{T}, \mathbb{G}_{E}\right)$ and $\mathbb{Q} \otimes D_{\infty}=C_{0}$. Ando constructs an Adams operation depending on $H$ as the composite

$$
\psi^{H}: E(X) \stackrel{P_{p} / I}{\longrightarrow} E\left(B \Sigma_{p}\right) / I \otimes_{E} E(X) \stackrel{H \otimes 1}{\longrightarrow} D_{\infty} \otimes_{E} E(X) .
$$

He then defines the $p^{k}$ th Hecke operator

$$
T_{p^{k}}=\sum_{\substack{H \subset \mathbb{T} \\|H|=p^{k}}} \psi^{H}
$$

and shows that this lands in $E(X)$.

\section{A canonical representative of the Frobenius class}

We construct a canonical representative of the set $[\bar{\sigma}]$. The construction is an elementary application of several of the tools presented in the previous section.

We specialize the transfers of the previous section to $G=\Sigma_{p}$. Let

$$
\operatorname{Tr}_{E}: E\left(B \Sigma_{p}\right) \rightarrow E
$$

be the transfer from $\Sigma_{p}$ to the trivial group and let

$$
\operatorname{Tr}_{C_{0}}: C l_{n}\left(\Sigma_{p}, C_{0}\right) \rightarrow C_{0}
$$

be the transfer in class functions from $\Sigma_{p}$ to the trivial group. This is given by the formula

$$
\operatorname{Tr}_{C_{0}}(f)=\frac{1}{p !} \sum_{\alpha} f(\alpha),
$$

where $\alpha \in \operatorname{hom}\left(\mathbb{Z}_{p}^{n}, \Sigma_{p}\right)$. There are $p^{n}$ elements in $\operatorname{hom}\left(\mathbb{Z}_{p}^{n}, \Sigma_{p}\right)$, so

$$
\operatorname{Tr}_{E}(1)=\frac{p^{n}}{p !}
$$


Recall that $\mathbb{T}=\left(\mathbb{Q}_{p} / \mathbb{Z}_{p}\right)^{n}$ and let $\operatorname{Sub}_{p}(\mathbb{T})$ be the set of subgroups of order $p$ in $\mathbb{T}$. The next lemma can be found in [BP03, Lemma 4.3, Lemma 5.1] for the cases of $B P$ and $K(n)$. The same methods give the calculation for Morava $E$-theory and, in this case, the result can be found in [Mar, Section 4.3.6].

Lemma 3.1 ([Mar, Section 4.3.6]). The restriction map along $\mathbb{Z} / p \subseteq \Sigma_{p}$ induces an isomorphism

$$
E\left(B \Sigma_{p}\right) \stackrel{\cong}{\longrightarrow} E(B \mathbb{Z} / p)^{\operatorname{Aut}(\mathbb{Z} / p)}
$$

After a choice of coordinate $x$,

$$
E\left(B \Sigma_{p}\right) \cong E[y] /(y f(y)),
$$

where the degree of $f(y)$ is

$$
\left|\operatorname{Sub}_{p}(\mathbb{T})\right|=\frac{p^{n}-1}{p-1}=\sum_{i=0}^{n-1} p^{i},
$$

$f(0)=p$, and $y$ maps to $x^{p-1}$ in $E(B \mathbb{Z} / p) \cong E \llbracket x \rrbracket /[p](x)$.

Lemma 3.2 ([Qui71, Proposition 4.2]). After choosing a coordinate, there is an isomorphism

$$
E\left(B \Sigma_{p}\right) / I \cong E[y] /(f(y)),
$$

and the ring is free of $\operatorname{rank}\left|\operatorname{Sub}_{p}(\mathbb{T})\right|$ as an E-module.

After choosing a coordinate, the restriction map $E\left(B \Sigma_{p}\right) \rightarrow E$ sends $y$ to 0 and the map

$$
E\left(B \Sigma_{p}\right) \rightarrow E\left(B \Sigma_{p}\right) / I
$$

is the quotient by the ideal generated by $f(y)$.

Lemma 3.3. The index of the $E$-module $E\left(B \Sigma_{p}\right)$ inside $E \times E\left(B \Sigma_{p}\right) / I$ is $p$.

Proof. This can be seen using the coordinate. There is a basis of $E\left(B \Sigma_{p}\right)$ given by the set $\left\{1, y, \ldots, y^{m}\right\}$, where $m=\left|\operatorname{Sub}_{p}(\mathbb{T})\right|$, and a basis of $E \times E\left(B \Sigma_{p}\right) / I$ given by

$$
\left\{(1,0),(0,1),(0, y), \ldots,\left(0, y^{m-1}\right)\right\} \text {. }
$$

By Lemma 3.1, the image of the elements $\left\{1, y, \ldots, y^{m-1}, p-f(y)\right\}$ in $E\left(B \Sigma_{p}\right)$ is the set

$$
\left\{(1,1),(0, y), \ldots,\left(0, y^{m-1}\right),(0, p)\right\}
$$

in $E \times E\left(B \Sigma_{p}\right) / I$. The image of $y^{m}$ is in the span of these elements and the submodule generated by these elements has index $p$.

Lemma 3.4 ([Rez09, Section 10.3]). In terms of a coordinate, the Frobenius class

$$
\bar{\sigma}: E\left(B \Sigma_{p}\right) / I \rightarrow E / p
$$

is the quotient by the ideal (y). 
Now we modify $\operatorname{Tr}_{C_{0}}$ to construct a map

$$
\sigma_{c a n}: E\left(B \Sigma_{p}\right) / I \rightarrow E .
$$

By Ganter's result [Gan06, Section 7.3] and the fact that $\Sigma_{p}$ is good, the restriction of $\operatorname{Tr}_{C_{0}}$ to $E\left(B \Sigma_{p}\right)$ is equal to $\operatorname{Tr}_{E}$. It makes sense to restrict $\operatorname{Tr}_{C_{0}}$ to

$$
E \times E\left(B \Sigma_{p}\right) / I \subset C l_{n}\left(\Sigma_{p}, C_{0}\right) .
$$

Lemma 3.3 implies that this lands in $\frac{1}{p} E$. Thus we see that the target of the map

$$
\left.p ! \operatorname{Tr}_{C_{0}}\right|_{E \times E\left(B \Sigma_{p}\right) / I}
$$

can be taken to be $E$. We may further restrict this map to the subring $E\left(B \Sigma_{p}\right) / I$ to get

$$
\left.p ! \operatorname{Tr}_{C_{0}}\right|_{E\left(B \Sigma_{p}\right) / I}: E\left(B \Sigma_{p}\right) / I \rightarrow E .
$$

From the formula for $\operatorname{Tr}_{C_{0}}$, for $e \in E \subset E\left(B \Sigma_{p}\right) / I$, we have

$$
\left.p ! \operatorname{Tr}_{C_{0}}\right|_{E\left(B \Sigma_{p}\right) / I}(e)=\left(p^{n}-1\right) e .
$$

Note that $p-1$ is a $p$-adic unit, so we may set

$$
\sigma_{c a n}=\left.\frac{p !}{p-1} \operatorname{Tr}_{C_{0}}\right|_{E\left(B \Sigma_{p}\right) / I} .
$$

There are several reasonable ways to normalize $\sigma_{c a n}$, we have chosen to divide by $p-1$ because there are $p-1$ maps in each nontrivial conjugacy class $\left[\alpha: \mathbb{Z}_{p}^{n} \rightarrow \Sigma_{p}\right]$. This normalization gives $\sigma_{c a n}(e)=\left|\operatorname{Sub}_{p}(\mathbb{T})\right| e$ for any $e \in E \subset E\left(B \Sigma_{p}\right) / I$. Another reason for this choice is explained in the next section.

We now show that $\sigma_{c a n}$ fits in the diagram

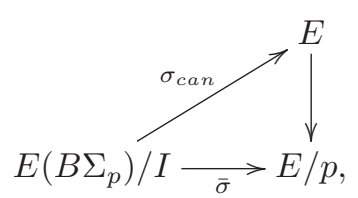

where $\bar{\sigma}$ picks out the kernel of the relative Frobenius.

Proposition 3.5. The map

$$
\sigma_{\text {can }}: E\left(B \Sigma_{p}\right) / I \rightarrow E
$$

is a representative of Rezk's Frobenius class.

Proof. We may be explicit. Choose a coordinate so that the quotient map

$$
q: E\left(B \Sigma_{p}\right) \rightarrow E\left(B \Sigma_{p}\right) / I
$$

is given by

$$
q: E[y] /(y f(y)) \rightarrow E[y] /(f(y))
$$

We must show that

$$
E\left(B \Sigma_{p}\right) / I \stackrel{\sigma_{c a n}}{\longrightarrow} E \stackrel{\bmod p}{\longrightarrow} E / p
$$

is the quotient by the ideal $(y) \subset E\left(B \Sigma_{p}\right) / I$. 
There is a basis of $E\left(B \Sigma_{p}\right)$ (as an $E$-module) given by $\left\{1, y, \ldots, y^{m}\right\}$, where $m=$ $\left|\operatorname{Sub}_{p}(\mathbb{T})\right|$. We will be careful to refer to the image of $y^{i}$ in $E\left(B \Sigma_{p}\right) / I$ as $q\left(y^{i}\right)$. For the basis elements of the form $y^{i}$, where $i \neq 0$, the restriction map $E\left(B \Sigma_{p}\right) \rightarrow E$ sends $y^{i}$ to 0 . Thus

$$
\operatorname{Tr}_{E}\left(y^{i}\right)=\left.\operatorname{Tr}_{C_{0}}\right|_{E\left(B \Sigma_{p}\right) / I}\left(q\left(y^{i}\right)\right) \in E .
$$

Now the definition of $\sigma_{c a n}$ implies that $\sigma_{c a n}\left(q\left(y^{i}\right)\right)$ is divisible by $p$. So

$$
\sigma_{\text {can }}\left(q\left(y^{i}\right)\right) \equiv 0 \bmod p .
$$

It is left to show that, for $e$ in the image of $E \rightarrow E\left(B \Sigma_{p}\right) / I$,

$$
\sigma_{\text {can }}(e) \equiv e \bmod p .
$$

Since $\sigma_{\text {can }}(e)=\left|\operatorname{Sub}_{p}(\mathbb{T})\right| e$, the result follows from the fact that $\left|\operatorname{Sub}_{p}(\mathbb{T})\right| \equiv 1 \bmod p$.

\section{The Hecke operator congruence}

We show that the $p$ th additive power operation composed with $\sigma_{c a n}$ is the $p$ th Hecke operator. This implies that the Hecke operator satisfies a certain congruence.

The two maps in question are the composite

$$
E(X) \stackrel{P_{p} / I}{\longrightarrow} E\left(B \Sigma_{p}\right) / I \otimes_{E} E(X) \stackrel{\sigma_{c a n} \otimes 1}{\longrightarrow} E(X)
$$

and the Hecke operator $T_{p}$ described in Section 2.

Proposition 4.1. The pth additive power operation composed with the canonical representative of the Frobenius class is equal to the pth Hecke operator:

$$
\left(\sigma_{\text {can }} \otimes 1\right)\left(P_{p} / I\right)=T_{p}
$$

Proof. This follows in a straight-forward way from the definitions. Unwrapping the definition of the character map, the map $\sigma_{c a n}$ is the sum of a collection of maps

$$
E\left(B \Sigma_{p}\right) / I \rightarrow C_{0},
$$

one for each subgroup of order $p$ in $\mathbb{T}$ (or non-trivial conjugacy class $\left[\alpha: \mathbb{Z}_{p}^{n} \rightarrow \Sigma_{p}\right]$ ).

Any map

$$
\alpha: \mathbb{Z}_{p}^{n} \rightarrow \Sigma_{p}
$$

factors through $(\mathbb{Z} / p)^{n}$. We will refer to the induced map $(\mathbb{Z} / p)^{n} \rightarrow \Sigma_{p}$ as $\alpha$ as well. Given $[\alpha]$, the character map to the factor of class functions corresponding to $[\alpha]$ is the composite

$$
E\left(B \Sigma_{p}\right) \stackrel{\alpha^{*}}{\longrightarrow} E\left(B(\mathbb{Z} / p)^{n}\right) \longrightarrow D_{\infty} \longrightarrow C_{0} .
$$

If $\alpha$ is surjective, then this induces

$$
E\left(B \Sigma_{p}\right) / I \stackrel{\alpha^{*}}{\longrightarrow} E\left(B(\mathbb{Z} / p)^{n}\right) / I_{t r} \longrightarrow D_{\infty} \longrightarrow C_{0},
$$

where $I_{t r} \subset E\left(B(\mathbb{Z} / p)^{n}\right)$ is the ideal generated by transfers from proper subgroups. It is standard that, as $[\alpha]$ varies, these are the maps that classify the subgroups of 
order $p$ in $\mathbb{T}$. For completeness, note that the subgroup can be read off of $[\alpha]$. It is image of the Pontryagin dual of the surjective map

$$
\alpha: \mathbb{Z}_{p}^{n} \rightarrow \operatorname{im} \alpha .
$$

Since $\sigma_{\text {can }} \in[\bar{\sigma}]$, the following diagram commutes

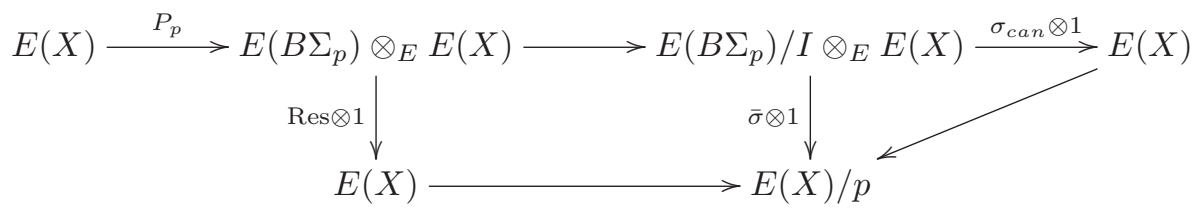

and this implies that

$$
\left(\sigma_{c a n} \otimes 1\right)\left(P_{p} / I\right)(x) \equiv x^{p} \bmod p .
$$

Corollary 4.2. For $x \in E(X)$, there is a congruence

$$
T_{p}(x) \equiv x^{p} \quad \bmod p .
$$

Let $X$ be a space with the property that $E(X)$ is torsion-free. The corollary above implies the existence of a canonical function

$$
\theta: E(X) \rightarrow E(X)
$$

such that

$$
T_{p}(x)=x^{p}+p \theta(x) .
$$

Example 4.3. When $n=1$ and $E$ is $p$-adic $K$-theory, $\mathbb{G}_{E}$ is a height 1 formal group,

$$
E\left(B \Sigma_{p}\right) / I
$$

is a rank one $E$-module, and $\sigma_{c a n}$ is an $E$-algebra isomorphism. The composite

$$
E(X) \stackrel{P_{p} / I}{\longrightarrow} E\left(B \Sigma_{p}\right) / I \otimes_{E} E(X) \stackrel{\sigma_{c a n} \otimes 1}{\longrightarrow} E(X)
$$

is the $p$ th unstable Adams operation. In this situation, the function $\theta$ is understood by work of Bousfield [Bou96].

Example 4.4. At arbitrary height, we may consider the effect of $T_{p}$ on $z \in \mathbb{Z}_{p} \subset E$. Since $T_{p}$ is a sum of ring maps

$$
T_{p}(z)=\left|\operatorname{Sub}_{p}(\mathbb{T})\right| z .
$$

This is congruent to $z^{p} \bmod p$.

Example 4.5. At height 2 and the prime 2, Rezk constructed an E-theory associated to a certain elliptic curve $[\mathbf{R e z}]$. He calculated $P_{2} / I$, when $X=*$. He found that, after choosing a particular coordinate $x$,

$$
E\left(B \Sigma_{2}\right) / I \cong \mathbb{Z}_{2} \llbracket u_{1} \rrbracket[x] /\left(x^{3}-u_{1} x-2\right)
$$

and

$$
P_{2} / I: \mathbb{Z}_{2} \llbracket u_{1} \rrbracket \rightarrow \mathbb{Z}_{2} \llbracket u_{1} \rrbracket[x] /\left(x^{3}-u_{1} x-2\right)
$$

sends $u_{1} \mapsto u_{1}^{2}+3 x-u_{1} x^{2}$. In [Dri74, Section 4B], Drinfeld explains how to compute 
the ring that corepresents $\mathbb{Z} / 2 \times \mathbb{Z} / 2$-level structures. Note that in the ring

$$
\mathbb{Z}_{2} \llbracket u_{1} \rrbracket[y, z] /\left(y^{3}-u_{1} y-2\right),
$$

$y$ is a root of $z^{3}-u_{1} z-2$ and

$$
\frac{z^{3}-u_{1} z-2}{z-y}=z^{2}+y z+y^{2}-u_{1}
$$

Drinfeld's construction gives

$$
D_{1}=\Gamma \operatorname{Level}\left(\mathbb{Z} / 2 \times \mathbb{Z} / 2, \mathbb{G}_{E}\right) \cong \mathbb{Z}_{2} \llbracket u_{1} \rrbracket[y, z] /\left(y^{3}-u_{1} y-2, z^{2}+y z+y^{2}-u_{1}\right) .
$$

The point of this construction is that $x^{3}-u_{1} x-2$ factors into linear terms over this ring. In fact,

$$
x^{3}-u_{1} x-2=(x-y)(x-z)(x+y+z) .
$$

The three maps $E\left(B \Sigma_{2}\right) / I \rightarrow D_{1} \subset C_{0}$ that show up in the character map are given by sending $x$ to these roots. We see that

$$
\sigma_{\text {can }}(x)=y+z-(y+z)=0
$$

and that

$$
\begin{aligned}
T_{p}\left(u_{1}\right) & =\left(\sigma_{\text {can }} \otimes 1\right)\left(P_{2} / I\right)\left(u_{1}\right) \\
& =3 u_{1}^{2}-2 u_{1} y^{2}-2 u_{1} z^{2}-2 u_{1} y z \\
& =u_{1}^{2}
\end{aligned}
$$

\section{References}

[AA66] J.F. Adams and M.F. Atiyah, $K$-theory and the Hopf invariant, Quart. J. Math. Oxford Ser. (2) 17 (1966), 31-38. MR 0198460 (33 \#6618).

[And95] M. Ando, Isogenies of formal group laws and power operations in the cohomology theories $E_{n}$, Duke Math. J. 79 (1995), no. 2, 423-485. MR 1344767 (97a:55006).

[Bou96] A.K. Bousfield, On $\lambda$-rings and the $K$-theory of infinite loop spaces, $K$ Theory 10 (1996), no. 1, 1-30. MR 1373816.

[BP03] M. Bakuradze and S. Priddy, Transfer and complex oriented cohomology rings, Algebr. Geom. Topol. 3 (2003), 473-509. MR 1997326

[Dri74] V.G. Drinfel'd, Elliptic modules, Mat. Sb. (N.S.) 94(136) (1974), 594627, 656. MR 0384707.

[Gan06] N. Ganter, Orbifold genera, product formulas and power operations, Adv. Math. 205 (2006), no. 1, 84-133. MR 2254309.

[GH04] P.G. Goerss and M.J. Hopkins, Moduli spaces of commutative ring spectra, Structured Ring Spectra, London Math. Soc. Lecture Note Ser., vol. 315, Cambridge Univ. Press, Cambridge, 2004, pp. 151-200. MR 2125040 (2006b:55010).

[GS96] J.P.C. Greenlees and Hal Sadofsky, The Tate spectrum of $v_{n}$-periodic complex oriented theories, Math. Z. 222 (1996), no. 3, 391-405. MR 1400199 (97d:55010). 
[HKR00] M.J. Hopkins, N.J. Kuhn, and D.C. Ravenel, Generalized group characters and complex oriented cohomology theories., J. Amer. Math. Soc. 13 (2000), no. 3, 553-594 (in English).

[Mar] S. Marsh, The Morava E-theories of finite general linear groups, http:/ /arxiv.org/abs/1001.1949.

[Qui71] D. Quillen, Elementary proofs of some results of cobordism theory using Steenrod operations, Adv. Math. 7 (1971), 29-56 (1971). MR 0290382.

[Rez] C. Rezk, Power operations for Morava E-theory of height 2 at the prime 2, arXiv:math.AT/0812.1320.

[Rez09] C. Rezk, The congruence criterion for power operations in Morava E-theory, Homology Homotopy Appl. 11 (2009), no. 2, 327-379. MR 2591924 (2011e:55021).

[Str98] N.P. Strickland, Morava E-theory of symmetric groups, Topology 37 (1998), no. 4, 757-779. MR 1607736 (99e:55008).

Nathaniel Stapleton nat.j.stapleton@uky.edu

Department of Mathematics, University of Kentucky, 719 Patterson Office Tower, Lexington, Kentucky 40506-0027, USA 